\title{
Epileptic Seizure Detection and Prediction Based on Continuous Cerebral Blood Flow Monitoring - a Review
}

\author{
Senay Tewolde ${ }^{1}$; Kalarickal Oommen, $\mathrm{MD}^{2}$; Donald Y. C. Lie, $\mathrm{PhD}^{3}$; Yuanlin \\ Zhang, $\mathrm{PhD}^{4}$ and Ming-Chien Chyu, $\mathbf{P h D}^{1, \mathbf{5}^{*}}$ \\ ${ }^{1}$ Department of Mechanical Engineering, Texas Tech University, Lubbock, TX \\ ${ }^{2}$ Covenant Comprehensive Epilepsy Center, Covenant Health System, Lubbock, Texas \\ ${ }^{3}$ Department of Electrical and Computer Engineering, \\ ${ }^{4}$ Department of Computer Science, \\ ${ }^{5}$ Graduate Healthcare Engineering, Texas Tech University, Lubbock, TX
}

Submitted January 2015. Accepted for publication April 2015

\begin{abstract}
Epilepsy is the third most common neurological illness, affecting $1 \%$ of the world's population. Despite advances in medicine, about 25 to $30 \%$ of the patients do not respond to or cannot tolerate the severe side effects of medical treatment, and surgery is not an option for the majority of patients with epilepsy. The objective of this article is to review the current state of research on seizure detection based on cerebral blood flow (CBF) data acquired by thermal diffusion flowmetry (TDF), and CBF-based seizure prediction. A discussion is provided on the applications, advantages, and disadvantages of TDF in detecting and localizing seizure foci, as well as its role in seizure prediction. Also presented are an overview of the present challenges and possible future research directions (along with methodological guidelines) of the CBF-based seizure detection and prediction methods.
\end{abstract}

Keywords: epilepsy, localization of seizure focus, cerebral perfusion, flowmetry

\section{INTRODUCTION}

Studies have shown that an excessive and abnormal synchronization of actively discharging neurons characterizes the dynamic process of human focal epilepsy $[1,2]$. The etiology of epilepsy in most cases remains unknown, and this may be in part due to the different types of seizures, symptoms, and intensities that seem to greatly vary from person to person [3]. Factors such as head injuries, damaged brain cells, brain tumor, brain chemical imbalances, febrile convulsions, genetic factors, and other developmental anomalies such as cerebral palsy are some of the associations noted where a cause is identifiable. Medication or surgery can lower the frequency and intensity of seizures or even make the patient seizure-free in about three-quarters of the

*Corresponding author: M. Chyu, Texas Tech University, Department of Mechanical Engineering Box 41021, Lubbock, Texas 79409-1021,USA.E-mail: m.chyu@ttu.edu.Phone: (806) 834 8982.E-mail: m.chyu@ ttu.edu. Other authors: senay.t.imam@ttu.edu; OOMMENKJ@covhs.org; Donald.lie@ttu.edu; y.zhang@ttu.edu. 
individuals diagnosed with the epilepsies [4]. However, approximately 25 to $30 \%$ of patients with epilepsy are refractory to medical therapy or have severe side effects of medication such as depression, suicidal thoughts and behaviors, and inflammation of certain organs. Surgical treatment is not an option if the seizure focus is not clearly identified or too vital to remove, if the patient has other serious medical problems (such as cancer or heart disease), or if lack of resources, complexity of the procedure, or high cost, particularly outside the developed countries, is an issue.

One of the most disabling aspects of epilepsy is the sudden occurrence of seizure in patients without an "aura" or warning. Inability of detecting an impending seizure has exposed epilepsy patients to risk of serious injury, intense feeling of helplessness, and social isolation [3]. Therefore, early detection and prediction of a seizure onset at a localized epileptic focus is critical for implementing appropriate preventive measures either to suppress the seizure or to warn the individual to seek medical attention or safe ground.

The techniques of localizing and detecting seizure onset have been continuously evolving from highly invasive brain surgery followed, in some cases, by electrical stimulation for functional mapping, to less invasive methods of functional brain mapping such as magnetic source imaging (MSI) or magnetoencephalography (MEG). Currently, electroencephalography (EEG) is considered the gold standard of monitoring the neural discharges during an epileptic seizure [5]. Several studies have reported the predictability of impending seizures by EEG using nonlinear and linear signal analysis techniques [5-9]. However, due to issues related to specificity, sensitivity to motion (such as subtle head movement), absence of reference period, and others [5, 10, 11], such techniques are yet to be established for reliable clinical application as tools for predicting seizure onsets.

Existing evidence in the literature suggests that monitoring local cerebral blood flow (CBF) may be a viable approach to early detection and eventually to the prediction of epileptic seizures. However, techniques and algorithms related to $\mathrm{CBF}$ and $\mathrm{CBF}$-based early detection and prediction of seizure onset are not available in the literature. Under normal circumstances, $\mathrm{CBF}$ in the two hemispheres is closely correlated under uniform vasomotor control; therefore, any stimulus that increases $\mathrm{CBF}$ causes bilateral $\mathrm{CBF}$ increase [12-17]. However, in the case of partial epilepsy, such as temporal lobe epilepsy, there is a regional $\mathrm{CBF}(\mathrm{rCBF})$ abnormality associated with the epileptogenic focus where cerebral perfusion between the two temporal lobes becomes inversely correlated during the inter-ictal and post-ictal periods [13-17]. Studies using long-term combined temporal lobe thermal diffusion flowmetry (TDF) and EEG showed that cerebral perfusion in the non-epileptic temporal lobe increases as the perfusion in the contralateral epileptic region decreases during these periods [13-17]. Normalization of the cerebral perfusion in these regions was observed during a 10 minute peri-ictal period [17].

CBF measurement devices such as thermal clearance (thermal diffusion flowmetry, TDF), neuroimaging modalities (single photon emission computed tomography, SPECT; positron emission tomography, PET; magnetic resonance imaging, MRI; stable xenonenhanced computed tomography, Xe/CT, etc.), Doppler techniques (transcranial Doppler 
sonography, TDS, laser Doppler flowmetry, LDF, etc.), and near infrared spectroscopy (NIRS) are also used as adjunctive modalities in monitoring, localizing and lateralizing epileptic seizure foci. However, applications of these methods for early seizure detection and prediction have not been developed due to their limitations in the duration of scanning, invasiveness (e.g., injecting and/or inhaling radioactive material such as ${ }^{133} \mathrm{Xe}$ and Xe [18], and implanting probes such as TDF and LDF [19, 20]), and extreme sensitiveness of signal to motion [21].

In addition, while all these techniques can provide information about $\mathrm{rCBF}$, most of them do not either provide a continuous measurement of CBF or quantify the change of $\mathrm{CBF}$ based on relative change. For instance, functional magnetic resonance imaging (fMRI) is known for its spatial resolution quality and soft tissue contrast, which made a significant contribution to the study of cortical activation during epileptic activity and defining the original activation area of the epileptogenic focus [22]. However, due to lack of high temporal resolution of fMRI, the exact relationship between surface EEG and blood oxygenation level dependent (BOLD) signal changes still remains unknown, and it is often impossible to differentiate the ongoing epileptic processes reflected on the BOLD from the surface EEG signals during EEG-fMRI scanning [23]. TDF is the only method that is capable of long-term, continuous, real-time, soft-tissue perfusion measurements in absolute values, and is considered one of the most feasible methods for the study of seizure detection and prediction through CBF monitoring.

This article reviews the current state of research on CBF-based seizure detection using TDF and mathematical methods of seizure prediction based on correlation between CBF change and impending seizure. Also presented are the present challenges in the methodological problems and pitfalls involved in the techniques for monitoring $\mathrm{CBF}$, and possible future research directions of CBF-based seizure detection and prediction methods.

\section{METHOD OF LITERATURE SEARCH}

Literature selection for this article was based on the PubMed and the Springer Link databases, and the Google Scholar search engine from 1933 to the present. The search was conducted using different combinations of the following terms: "epilepsy", "detection", "prediction", "seizure onset", "localization", "seizure focus", "CBF", "flowmetry", and "EEG". Articles that were considered to be pertinent to this review and written in English were included.

\section{CEREBRAL BLOOD FLOW MEASUREMENT BY THERMAL DIFFUSION FLOWMETRY (TDF)}

TDF is one of the most common methods for monitoring $\mathrm{rCBF}[21,24,25]$. It is widely used for long-term bedside monitoring of $\mathrm{rCBF}$ in comatose patients [26], patients with brain injuries [20], and patients during and after surgery [21, 25, 27, 28]. The rCBF is determined by measuring the effective thermal conductivity of the cortical tissue, which changes with $\mathrm{rCBF}$, using a temperature microprobe implanted in the cerebral region of interest (ROI). It measures dynamic changes in cerebral perfusion by converting the value of temperature gradient to $\mathrm{ml}$ of blood per $100 \mathrm{~g}$ of brain tissue per min, $\mathrm{ml} /$ (100g-min), in real-time [19, 20, 29-32]. 

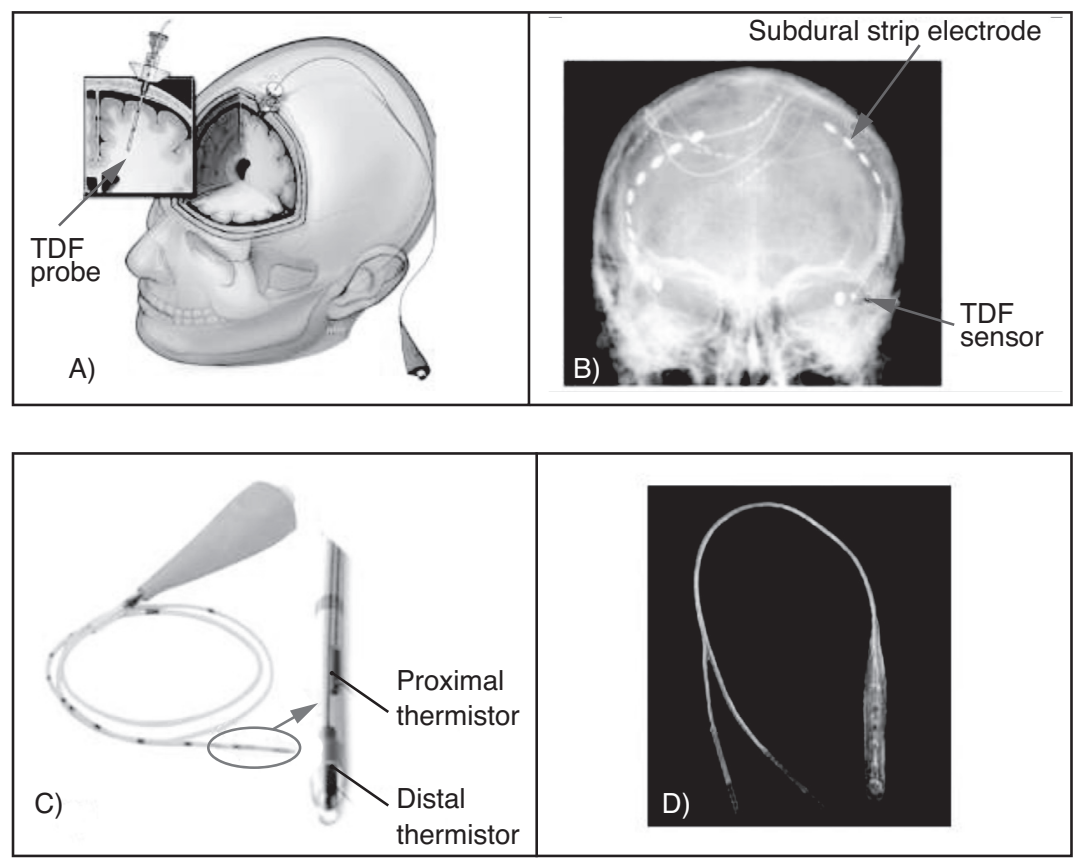

Figure 1. Thermal diffusion flowmetry (TDF) probes for $\mathrm{CBF}$ measurement. (A) and (B) illustrate installation of the TDF probes shown in (C) and (D), respectively [13, 20,33]. Reproduced with permission.

TDF probe typically monitors a small area of the brain cortex either by inserting the probe into the targeted soft tissue (below the dura) through a small burr hole (Figure 1A) $[20,33]$ or craniotomy in the most superficial 2 to $3 \mathrm{~mm}$ of the cortex $[19,28,31]$, or by installing it on the cortical surface of the ROI (Figure 1B) [13].

Figure 1A [20, 33] depicts installation of a commercial TDF, QFlow 500 Perfusion Probe (Hemedex, Cambridge, MA), as shown in Figure 1C [33], inserted into the soft tissue. The probe has two sensors, one near the tip (viz. distal thermistor), that measures heated tissue temperature, and the other located $8 \mathrm{~mm}$ proximal to the tip (viz. proximal thermistor), that measures the baseline (unheated) tissue temperature. Both thermistors are housed in a 0.9-mm-diameter polyurethane catheter (Figure 1C) $[20,33]$. The distal thermistor is heated to approximately $2^{\circ} \mathrm{C}$ above the baseline tissue temperature, generating a constant (with respect to time) spherical thermal field of about 4-mm diameter $[20,33,34]$. The QFlow $500^{\mathrm{TM}}$ Probe is connected to a monitor (Bowman Perfusion Monitor, Hemedex, Cambridge, MA), as shown in Figure 2A, via an umbilical cord [33].

The other type of TDF features installation on the cortical surface of the ROI, as illustrated in Figure 1B [13-15], using another commercial thermal diffusion probe (Flowtronics, Phoenix, AZ) shown in Figure 1D. In some studies, TDF sensors are 
A

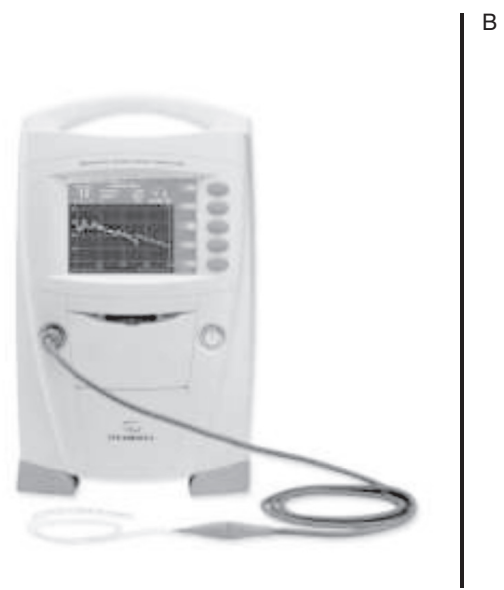

$\mathrm{B}$

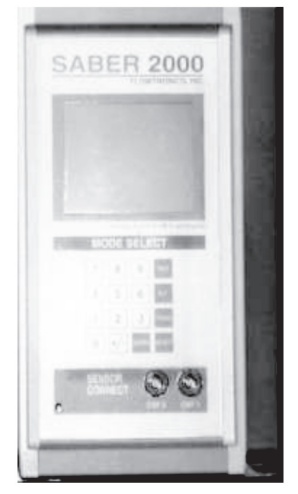

Figure 2. TDF monitoring devices. (A) Bowman Perfusion Monitor, and (B) Saber 2000. Reproduced with permission.

coupled with EEG subdural strip electrodes which detect clinical seizure onset, as shown in Figure 1B [13-15]. The probe shown in Figure 1D consists of two small thermistors (neutral and heated) of 24-k gold plates, and monitors the CBF using Saber 2000 Monitor (Flowtronics, Phoenix, AZ), as shown in Figure 2B [36]. The heated sensor measures a cortical area of approximately $1 \mathrm{~cm}$ deep in the cerebral hemisphere and $3 \mathrm{~cm}$ by implication [36]. Bowman Perfusion Monitor and Saber 2000 are the only two bedside devices commercially available for CBF recording at present.

The continuous transfer of thermal energy between the sensor and the tissue in contact can be monitored in either constant heat generation rate (isocaloric) or constant temperature (isothermal) fashion. In the isocaloric type of TDF, one sensor serves as both the temperature sensor and the heat source with constant heat generation rate (heating power), while the other compensates for the baseline temperature change $[19,51]$. The temperature measured by the heated sensor (i.e., the perfusion sensor) provides a continuous measurement of the effective thermal conductivity of the perfused tissue, which is directly related to the CBF [19, 20, 29, 35, 37]. In the isothermal type of TDF, power is supplied to one of the two thermistors in order to maintain a constant volumetric mean temperature difference between the heated and the unheated thermistors $[19,37,41-45]$, and such power provides a measurement of the effective thermal conductivity of the perfused tissue and thus the CBF.

The current review presents the first summary of theory published in literature leading to the clinical application of commercial TDF devices. Several studies have analyzed the nonlinear relationship between the electric heating power and blood perfusion [35, 37-47] using the bioheat transfer equation introduced by Pennes [48]. For both isocaloric and isothermal arrangements, assuming that the thermistor is a spherical bead with a radius $R_{s}$ (Figure 3), self-heated (with internal heat generation), and in perfect thermal contact with the surrounding tissue, and no major vessels in the thermal 


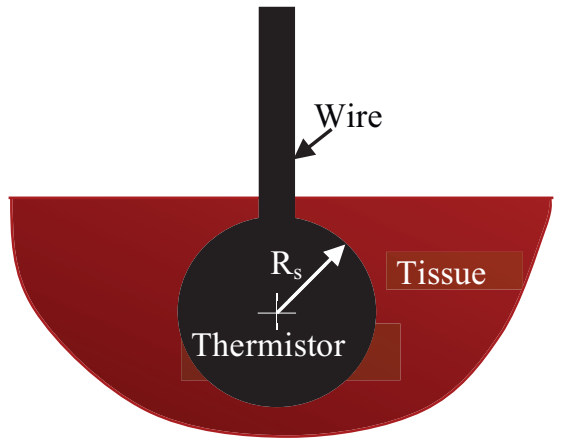

Figure 3. A thermistor bead in a semi-infinite domain of cortical tissue.

field of influence around the thermistor, the heat transfer equations for a thermistor embedded in a perfused tissue based on a coupled thermal model [35-47] are:

$$
\begin{gathered}
\rho_{t} c_{t} \frac{\partial T_{t}}{\partial t}=k_{t} \nabla^{2} T_{t}+\rho_{b} c_{b} \omega_{b}\left(T_{c}-T_{t}\right), \quad r>R_{s} \\
\rho_{s} c_{s} \frac{\partial T_{s}}{\partial t}=k_{s} \nabla^{2} T_{s}+g_{\text {ext }}(t), \quad r<R_{s} \\
g_{\text {ext }}(t)=\frac{\Gamma+\beta f(t)}{V_{s}}=\frac{P(t)}{V_{s}}
\end{gathered}
$$

where $\rho_{t}, \rho_{b}$, and $\rho_{s}$ are the densities of the tissue, blood, and the thermistor (sensor), respectively; $T_{t}, T_{c}$ and $T_{s}$ are the tissue temperature, core-body temperature, and the thermistor temperature, respectively; $c_{t}, c_{b}$ and $c_{s}$ are the specific heats of the tissue, blood, and the thermistor, respectively; $t$ is time; $k_{t}$ and $k_{s}$ are the thermal conductivities of the tissue structure (without liquid) and the thermistor, respectively; $\omega_{b}$ is the perfusion rate of the blood; and $r$ is the radial distance from the center of the thermistor. Note that eqn. 1 assumes that the metabolic heat generation rate can be ignored if it is much smaller than the volumetric heat generation rate from the thermistor [35], and that the blood is at the core-body temperature $T_{c}$. The volumetric heat generation rate within the probe, $g_{\text {ext }}(t)$ is separated into a steady-state power term, $\Gamma$, and a transient power function, $\beta f(t)$ [35, 41, 42]; collectively, they are represented by $P(t)$, the power to heat the sensor bead [41-43].

For the initial condition, $T_{s}$ and $T_{t}$ are assumed to be at the core-body temperature $\left(T_{c}\right)$ :

$$
T_{t}=T_{c} \text { and } T_{s}=T_{c}, \quad t=0
$$


The boundary conditions are no heat flux at the center of the thermistor due to symmetry,

$$
\frac{d T_{s}}{d r}=0, \quad r=0,
$$

and the same temperature and heat flux at the tissue-thermistor interface [37],

$$
\begin{gathered}
T_{s}=T_{t}, \quad r=R_{s} \\
-k_{s} \frac{\partial T_{s}}{\partial n}=k_{t} \frac{\partial T_{t}}{\partial n}, \quad r=R_{s}
\end{gathered}
$$

where $\boldsymbol{n}$ is the unit vector normal to the surface of the thermistor. The tissue far away is undisturbed by the thermistor and thus remains at the same temperature as the core-body temperature [37]:

$$
\lim _{r \rightarrow \infty} T_{t}=T_{c}
$$

In general, to measure tissue perfusion, thermal conductivity and/or thermal diffusion, the relationship between the applied thermistor power, $P(t)$, and the volumetric mean temperature difference between the heated and the unheated thermistors, $\Delta \bar{T}_{s}(t)$, have to be known. In the isocaloric method, $P$ is held constant while $\Delta \bar{T}_{s}$ changes with time, and the transient solution for $\Delta \bar{T}_{s}$ as a function of perfusion and intrinsic thermal properties of the tissue structure (excluding fluid) is given by [34, 37]:

$$
\begin{gathered}
P(t)=P_{o}=\frac{4}{3} \pi R_{s}^{3} g_{\text {ext }} \\
\Delta \bar{T}_{s}(t)=\frac{P_{o}}{4 \pi R_{s} k_{t}}\left[\frac{k_{t}}{5 k_{s}}+\frac{1}{1+\lambda R_{s}}-\frac{R_{s} / \sqrt{\frac{\pi k_{t}}{\rho_{t} c_{t}}}}{1-\lambda^{2} R_{s}^{2}} f(t)\right] \\
f(t)=\frac{e^{-\frac{\lambda^{2} k_{t} t}{\rho_{t} t_{t}}}}{\sqrt{t}}-\lambda \sqrt{\frac{\pi k_{t}}{\rho_{t} c_{t}}} \operatorname{erfc}\left(\lambda \sqrt{\frac{k_{t} t}{\rho_{t} c_{t}}}\right) \\
\lambda=\sqrt{\frac{\omega_{b} \rho_{b} c_{b}}{k_{t}}}
\end{gathered}
$$

where $P_{o}$ is the constant power supplied to the thermistor bead.

In the isothermal type of TDF, power is supplied to maintain a constant volumetric mean temperature difference $\Delta \bar{T}_{s}(t)$ between the heated and the unheated thermistors 


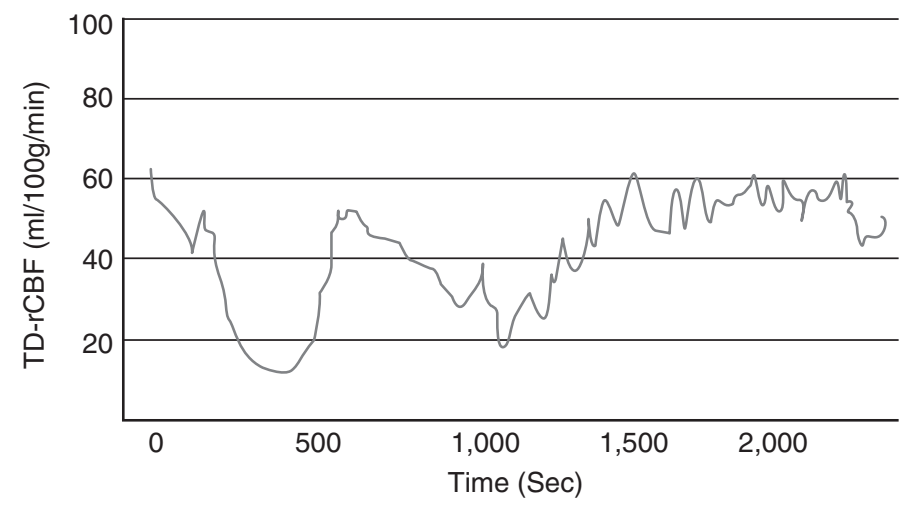

Figure 4. Sample of clinical CBF data [20]. Reproduced with permission.

$[19,37,41-45]$. The required heating power to the thermistor bead, $P(t)$ and the transient temperature field of the surrounding tissue can be solved by Laplace transformation (see details in $[40,41,43,44])$. The thermistor-tissue heat equations and the boundary conditions are formulated in the same manner as eqns. $1-7$. The solutions for the power distribution and temperature change are the following [37, 42]:

$$
\begin{gathered}
P(t)=\frac{4 \pi R_{s} k_{t} \Delta T_{o}}{\frac{k_{t}}{5 k_{s}}+\frac{1}{1+\lambda R_{s}}}\left[1+\frac{R_{s}}{\sqrt{\frac{\pi k_{t}}{\rho_{t} c_{t}}}\left(\frac{k_{t}}{5 k_{s}}\left(1-\lambda^{2} R_{s}^{2}\right)+1+\lambda R_{s}\right)} f(t)\right] \\
\Delta \bar{T}_{s}(t)=\frac{1}{V_{s}} \int_{V_{s}} \Delta T_{s}(r, t) d V_{s}=\frac{3}{4 \pi R_{s}^{3}} \int_{V_{s}} \Delta T_{s}(r, t) d V_{s}=\Delta T_{o}
\end{gathered}
$$

where $\Delta T_{o}$ is the constant temperature difference maintained between the two thermistor sensors. Figure 4 shows the continuous measurement of $\mathrm{CBF}$ by an isothermal TDF [20]. The recording was generated using a QFlow 500 ${ }^{\mathrm{TM}}$ Perfusion Probe (Figure 1C) and a Bowman Perfusion Monitor (Figure 2A), where the distal thermistor is heated to approximately $2^{\circ} \mathrm{C}$ above the tissue temperature $\left(T_{c}\right)$, generating a spherical temperature field of about $4 \mathrm{~mm}$ diameter [20].

To determine the thermal conductivity of the tissue, assuming no perfusion and steady state condition, eqn. 13 is substituted into the steady state equation of power, $P$ (eqn. 12 with $f(t)=0)[35,41,45]$, and $k_{t}$ is solved as:

$$
k_{t}=\frac{1}{\frac{4 \pi R_{s} \Delta \bar{T}_{s}}{\Gamma}-\frac{1}{5 k_{s}}}
$$

Calibration of TDF was performed using functional form of $k_{t}$ solution, eqn. 14 [35, 45]: 


$$
k_{t}=\frac{1}{\left(C_{1} \frac{\Delta \bar{T}_{s}}{\Gamma}\right)+C_{2}}
$$

with the coefficients $C_{1}$ and $C_{2}$ empirically determined by thermistor measurement of known thermal conductivities such as those of glycerol and agar-gelled water [45]. Comparison of the theoretical and the empirical expressions of $k_{t}$ (eqns. 14 and 15) shows that $C_{1}$ is a function of the geometry of the thermistor bead, and $C_{2}$ is a function of the thermistor's thermal conductivity $1 / 5 k_{s}$.

The other calibration necessary is to establish the relationship between the thermistor temperature measurement and its electrical resistance, using the following equation $[35,45]$ :

$$
T_{s}=\frac{1}{H_{o}+H_{1} \ln \left(\Phi_{s}\right)+H_{3}\left[\ln \left(\Phi_{s}\right)\right]^{3}}-273.15
$$

where $T_{s}$ is the temperature measured by the thermistor in ${ }^{\circ} \mathrm{C}, \Phi_{s}$ is the electrical resistance of the thermistor, and $H_{o}, H_{1}$ and $H_{3}$ are calibration coefficients determined empirically by experiment and nonlinear regression.

Finally, to estimate the steady state response, a measure of the thermal conductivity, Valvano et al. [45] measured the applied heating power, $P(t)$, and determined through linear regression the steady state response using the following correlation [35]:

$$
\frac{P(t)}{\Delta T}=I+S t^{-1 / 2}
$$

where $I$ is the coefficient of steady state response that depends on the thermal conductivity of the medium, and $S$ is the coefficient of transient response (slope) that depends on the thermal diffusivity of the medium $[35,46]$. Figure 5 shows such transient response of a TDF probe submerged/embedded in glycerol, tissue and agar-gelled water, respectively, to determine $I$ and $S$ [45]. The above correlation was also adopted by Balasubramanium [44], and Patel [46] for the same purpose.

Information for additional calibrations such as the relationship between analog to digital converter sample and the thermistor resistance, and the empirical correlation between effective thermal conductivity and perfusion, can be found in Chato [40], Valvano et al. [41, 45], Bowman et al. [37, 43], and Patel et al. [46].

\section{PREVIOUS EPILEPSY STUDIES USING TDF}

Prior to the early 1930s, observations of the CBF changes were conducted visually under craniotomy where seizures were triggered by electrical stimulation which resulted in visible pulsation of the cortex. For instance, Foerster [50] performed an extensive electrical stimulation on an exposed cerebral cortex of more than 100 patients under local anesthesia. In 1933, Penfield reported a detailed clinical study on the changes of the blood flow during and after seizure observed through craniotomy in 


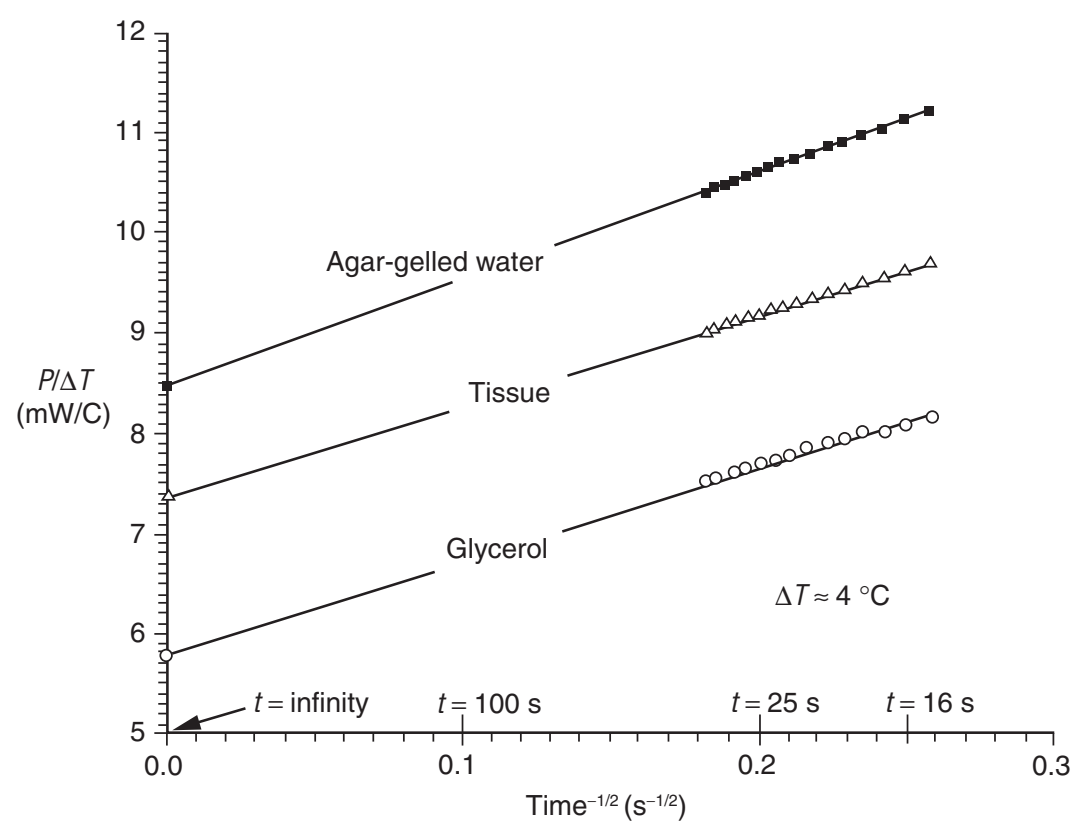

Figure 5. Regression plot to determine the steady state response of a thermistor based on transient heating power applied and constant temperature (isothermal) heating technique [45]. Reproduced with permission.

43 patients treated with electrical stimulation in the brain to trigger seizure. According to his report, during a seizure, arterial constriction (i.e., pulsation in the arteries of the brain stopped temporarily and resumed violently moments after the attack), areas of anemia, and other vasomotor manifestation appeared to occur [51]. The study suggested that under normal conditions, cerebral blood circulation occurs locally in the areas where the nerve cells are functionally active; however, no explanation is provided on the role of ischemia and the altered circulation in relation to the epileptic seizure [51].

Gibbs revolutionized the study of CBF by introducing TDF using modified thermocouples [52]. He reported the instrument's response to changes in blood flow through a series of experiments on parietal cortex of animals and internal jugular vein of human subjects [52]. His contribution has been celebrated as one of the greatest achievements in clinical epileptology, and his method continues to be employed by modern clinicians and researchers for long-term CBF monitoring.

Using the TDF device, in 1934, Gibbs et al. investigated for the first time CBF in epileptic patients and presented a continuous record of the changes in the flow of cerebral blood before a spontaneous seizure occurred [53]. Subsequently in 1939, applying Gibbs' technique, Penfield observed CBF changes in monkeys, cats and humans before seizure onsets [54]. Both studies [53, 54] found no evidence of change in CBF preceding an epileptic attack. In 1971, based on his previous observations through craniotomy, Penfield hypothesized that epileptic lesions of all types show cytological evidence of preceding progressive small ischemia [55]. In 1973, Carter and Atkinson further modified Brawley's 


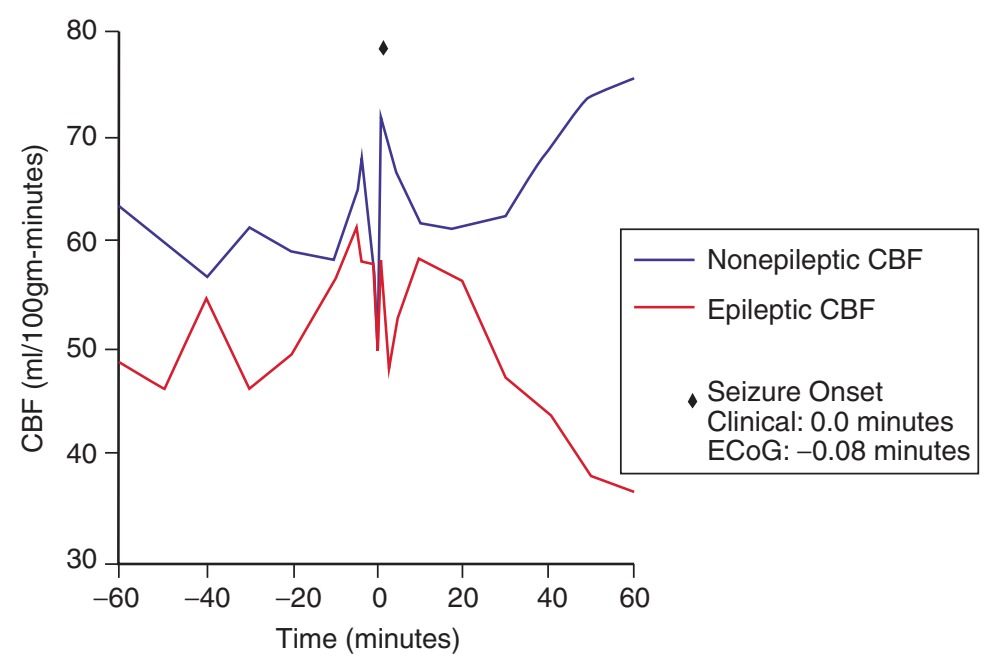

Figure 6. Variation of the mean CBF of 13 patients in non-epileptic and epileptic temporal lobes before, during, and after a seizure [14]. Reproduced with permission.

Peltier thermal diffusion probe [56] and calibrated the probe with ${ }^{133} \mathrm{Xe}$ clearance in cats [57]. They further developed a mathematical model that describes the quantification of $\mathrm{CBF}$ based on the linear correlation of temperature gradient and fast component of ${ }^{133 \mathrm{Xe}}$ clearance [19, 57-59]. In 1997, Weinand et al. observed significant changes of CBF in both epileptic and nonepileptic regions of a human brain about 10 minutes prior to the seizure onset [14]. Figure 6 shows their result based on CBF variations of 13 patients in both regions before, during, and after a seizure. CBF in the epileptic region is inversely correlated with that of the normal (non-epileptic) region during the inter-ictal and post-ictal periods, and normalizes during the peri-ictal period. This is a reflection of the increase in the blood flow in the epileptogenic focus, which of necessity must equalize or exceed the normal side as a result of the rise in metabolism. The subsequent studies by Weinand et al. further showed that if the CBF in the epileptic region increases and approximates the $\mathrm{CBF}$ in the nonepileptic region, seizure interval increases [15-17]. These results suggested an important role that epileptic cortical perfusion plays in temporal lobe epileptogenicity, lending credence to Penfield's hypothesis that epileptic seizure is the result of progressive small ischemias [55]. However, it is still unclear whether the early pre-ictal CBF changes are due to redistribution of blood from non-epileptic to epileptic cortex, and if such changes influence epileptogenicity $[14,15]$. Table 1 summarizes the methods for detection of seizures through CBF since Penfield's early observations of CBF changes during seizures.

Despite its capability to detect seizure or even to predict seizure prior to onset, TDF has not seen significant clinical use in the study of epilepsy. The last quantitative analysis of CBF data measured using TDF was published in 2004 by Gonzalez-Portillo, et al. [17] where they used the CBF data recorded by Weinand et al. [14] to verify the correlation between the epileptic and non-epileptic temporal lobes suggested by 
Table 1. Summary of studies on seizure detection and localization through CBF measurement by TDF

\begin{tabular}{lll}
\hline Authors & Year & \\
\hline Penfield [51] & $1933 \begin{array}{l}\text { Craniotomy in epilepsy patients showed very little blood } \\
\text { passing through the capillary bed of the brain during the } \\
\text { actual seizure. }\end{array}$ \\
Gibbs et al. [53] & $1934 \begin{array}{l}\text { First to use TDF technique to measure blood flow in internal } \\
\text { jugular vein of epileptic patients and to show continuous } \\
\text { changes in the blood flow before a spontaneous seizure. }\end{array}$ \\
& $1939 \begin{array}{l}\text { Found no evidence of decreased blood flow preceding an attack. } \\
\text { Observed an increase in CBF both in animals and } \\
\text { epilepsy patients during seizure within the seizure focus. } \\
\text { Penfield [54] }\end{array}$ \\
A decrease or no alteration in CBF was also observed in \\
epilepsy patients' cortical areas far from the focus.
\end{tabular}

Weinand et al. $[14,15]$ (Table 1). The paucity of TDF data might be due to the level of invasiveness of the procedure, and its ability to measure the CBF only in the ROI. There was a concern that the reliability of this technique is critically dependent on the precise positioning of the probe with respect to the seizure focus $[17,25]$. 


\section{COMPARISON OF EPILEPSY STUDIES USING TDF WITH OTHER STUDIES}

A result similar to those of the epilepsy studies using TDF reviewed above was reported by Baumgartner et al. in 1998 using Single Photon Emission Computed Tomography (SPECT), about an increase in blood perfusion in the epileptic temporal lobe approximately 12 minutes before the seizure onset [1]. Federico et al. reported a similar result using fMRI for three patients with refractory partial epilepsy, showing major bold-oxygen-level dependence (BOLD) signal changes several minutes prior to the seizure onset in areas that include, but not limited to, the seizure focus [61].

Further, Oommen et al. [62] demonstrated the dynamism of the peri-ictal CBF in 46 patients using video-EEG and monitored inter-ictal, peri-ictal, and immediately postictal CBF with SPECT, which corroborated the observation they reported in 1997 using TDF probes [14]. More recently, Oommen et al. reported changes of CBF in a rat monitored by EEG and laser Doppler flowmetry (LDF), demonstrating an increase in cerebral perfusion with the onset of the epileptiform electrical discharge and CBF restoring to the basal level within several minutes after the seizure [63]. The study provided an independent confirmation of the changes in CBF related to epileptic seizure observed by Weinand et al. [14]. In part, the study also provided further validation of Penfield's hypothesis [55].

\section{CBF-BASED SEIZURE PREDICTION}

A reliable method of seizure prediction is highly desirable, as it can afford appropriate prevention and suppression measures such as pre-emptive injection or local shock therapy $[6,64]$, warning the individual to seek medical attention or a safe harbor, and alerting medical personnel of the patient's impending seizure. In addition, seizure prediction algorithms can help medical personnel take appropriate actions by providing continuous feedbacks or seizure frequency. Most patients and caregivers preferred 3 to 5 minutes of warning time of an impending seizure [65].

No mathematical model has been published to predict seizure onset based on the dynamic change of $\mathrm{CBF}$, despite the existing evidence of $\mathrm{CBF}$ perturbation prior to a seizure onset. One of the major barriers to the development of such methods, both in terms of verification (to build the thing right) and validation (to build the right thing), is the paucity of clinical data. High quality long-term continuous CBF recordings from an adequate number of patients, with different seizure types, are required to develop and test a reliable seizure prediction algorithm. On the other hand, a number of publications have presented different mathematical methods for detection and prediction of a seizure using EEG data; however, no algorithm is widely accepted for such application [5, 6, 66, 67-71]. More research is still needed to improve the mathematical and statistical analysis of the EEG-based prediction methods [70,71].

Almost all of the published studies on seizure prediction are based on (a) retrospective analyses of EEG data, where sampled pre-seizure and baseline data are compared to reveal statistically significant differences associated with impending seizures, post facto, or (b) quasi-prospective analysis, where the algorithm rely on past information $[3,6,10,11,64$, 68-71], with the exception of a recent study published by Cook et al. [8]. For the first time, 
they presented clinical results of prospective seizure prediction of 15 patients, with 11 patients meeting the performance criteria of their prediction algorithm. In addition, they reported that their prediction technique performs better than chance [8], although it is not yet clinically applicable.

In general, prediction methodology for EEG can be adapted to predict seizure using CBF data with minor modifications in the prediction criteria and statistical testing. Methods of nonlinear dynamics, different types of complexity measures and the like can be applied to analyze CBF changes and to extract characteristic features that are predictive of seizure onset. Similar statistical frameworks described by Schelter et al. [7], Snyder et al. [70], and Winterhalder et al. [72] showcase the performance and the likelihood of success of the prediction algorithm. Possible application of these mathematical approaches in CBF data analysis is elaborated below.

\subsection{Time Domain Analysis}

Time domain analysis is a part of time series analysis used in analyzing stochastic signals. The analysis often involves comparison of two different signals that are statistically correlated. In predicting seizure through CBF changes, the relationship between the cortical epileptic and non-epileptic CBF can be compared statistically, which in turn is related to either an impending seizure or the inter-seizure interval. In addition, the time series analysis can be applied to identify the characteristic features (patterns) of the CBF in both the epileptic and the non-epileptic sides during the periictal state. Such pattern, however, requires CBF recordings over multiple seizure onsets. The recordings could be a univariate time series, where a single observation is recorded sequentially over multiple seizure intervals.

Weinand et al. [14-16] and Gonzalez-Portillo et al. [17] demonstrated the predictability of seizure by elucidating the relationship of $\mathrm{CBF}$ change between the epileptic and non-epileptic regions during the pre-ictal period, as well as the relationship between seizure intervals and CBF variation in the two regions. However, no seizure prediction method/algorithm was established.

\subsection{Linear/Nonlinear Dynamics}

The linear and/or nonlinear phenomena of the blood flow associated with a seizure can be determined from continuous CBF recordings. As mentioned in sections 4 and 6, a number of studies have shown that $\mathrm{CBF}$ changes minutes prior to a seizure onset. In order to characterize the dynamical behavior of CBF during such state, the pathological activity in the CBF data need to be quantified using a variety of univariate and bivariate linear and/or nonlinear time-based measures. Depending on the behavior of the data, these measures are expected to extract characteristic features that are predictive of seizure onset. For instance, applying the concept of entropy, such as approximate entropy, sample entropy and spectral entropy, can quantify the complexity levels of the epileptic and normal CBF patterns, and can provide evidence of state changes (i.e., preictal and inter-ictal) leading to seizure onset. Other methods such as autoregressive (AR) analysis, and various neural network models used in long-term EEG signal analysis may also be adapted for early detection of CBF changes. 


\subsection{Statistics of Seizure Warning}

The statistics of EEG-based seizure warning system is a widely studied topic of seizure prediction; however, evaluating the prediction success rate is still a statistical challenge [70]. Although statistical methods of seizure prediction are well documented [8-10, 70,72], formal validation or benchmark against chance predictor is yet to be established [11]. For a seizure warning/prediction system to be of clinical application value, the algorithm should exhibit at least acceptable sensitivity and specificity. To address the issue of how many false and missed predictions can be tolerated by patients, a survey shows that most patients expect a device to be able to successfully predict at least $90 \%$ of the seizure occurrences, and most of these patients can tolerate a considerable number of false predictions as long as the device can make fewer false predictions than correct predictions [73].

A similar statistical validation technique can be developed for CBF-based prediction algorithms. The correlation of the $\mathrm{CBF}$ between the epileptic and non-epileptic regions of the brain has been documented; however, developing a validation method or benchmark requires a statistical analysis based on a long-term continuous clinical CBF data. For example, to verify a false prediction rate of one false alarm per day would require at least 24 hours of interictal data, in which only one false prediction would be permitted [72].

\section{PRESENT CHALLENGES}

In order to develop a reliable seizure prediction algorithm based on a clear understanding of the physiological mechanism of CBF changes before seizure onset, a large archive of continuous epileptic CBF data is needed. Collecting such epileptic $\mathrm{CBF}$ data requires consents from a large number of patients to participate in data acquisition and clinical studies involving invasive procedure for a considerable length of time. There is certainly a great deal of concern among epileptic patients toward invasive procedures and implantable devices [65]. The most recent data were 43 2-hour continuous CBF recordings from 13 patients published in 1997 by Weinand et al. [14]. These concerns may be addressed through education, open discussion about the safety and risks, and lowering the cost of implementing and maintaining device [65].

\section{OUTLOOK AND FUTURE DIRECTIONS}

Despite the continuous advancement in neuroimaging technology which may bring forth a powerful imaging modality suitable for continuous CBF monitoring, at present, TDF seems to be one of the most reliable and suitable devices for long-term continuous $\mathrm{CBF}$ monitoring. Therefore, feasibility of CBF-based seizure prediction may mainly rely on the clinical application of TDF.

A key issue in seizure prediction based on CBF is the ability to identify the transition from an inter-ictal period to a pre-ictal period $[3,14]$. A reliable algorithm is required that can analyze the CBF pattern and predict and issue a warning prior to a seizure onset while identifying all the confounding physiological variables that may compromise the performance of the algorithm. 
One of the greatest challenges of seizure prediction by EEG is the difficulty in demonstrating that the prediction technique is significantly better than chance $[6,74]$. A similar challenge is expected for a CBF-based seizure prediction algorithm. The reliability and accuracy of a prediction algorithm will need to be validated in long-term clinical studies. Such algorithm will not only detect and anticipate a seizure onset but also help incorporate a more effective and acute treatment measures.

\section{CONCLUSION}

The existing evidence in the literature suggests that monitoring CBF may be a viable approach to detection and prediction of epileptic seizures. Detection of seizure onset using TDF and neuroimaging modalities such as fMRI has been documented; however, no study has demonstrated that seizure onset can be reliably predicted by dynamic changes of $\mathrm{CBF}$ in the pre-ictal stage. The present review leads to the following recommendations with respect to CBF-based seizure prediction:

1. CBF data of high temporal resolution should be collected from an at least adequate sample of epileptic patients with medically intractable seizures, providing details in CBF pattern's variation during the transition from the interictal to the pre-ictal period.

2. A method to predict seizure onset may be developed by quantifying the degree of complexity of the CBF pattern that can provide evidence of state changes leading to seizure onset, while taking into account all the confounding variables.

3. The seizure prediction method should demonstrate, in prospective clinical studies, acceptable sensitivity, specificity, and sufficient warning time prior to seizure onset, with a high margin of prediction confidence interval.

\section{CONFLICT OF INTEREST}

The authors indicated no potential conflicts of interest.

\section{NOMENCLATURE}

$\begin{array}{cl}C_{1} \text { and } C_{2} & \text { Coefficients } \\ c_{b} & \text { Specific heat of the blood, } \mathrm{J} /(\mathrm{kg}-\mathrm{K}) \\ c_{t} & \text { Specific heat of the tissue, } \mathrm{J} /(\mathrm{kg}-\mathrm{K}) \\ c_{s} & \text { Specific heat of the thermistor, } \mathrm{J} /(\mathrm{kg}-\mathrm{K}) \\ \mathrm{CBF} & \text { Cerebral blood flow } \\ \mathrm{CT} & \text { Computed tomography } \\ \text { EEG } & \text { Electroencephalography } \\ f(t) & \text { Transient power function, } \mathrm{s}^{-1 / 2} \\ g_{\text {ext }} & \text { Volumetric heat generation rate in the thermistor, } \mathrm{W} / \mathrm{m}^{3} \\ H_{o}, H_{1} \text { and } H_{3} & \text { Calibration coefficient } \\ I & \text { Steady state response, depending on the thermal conductivity, W/K } \\ k_{t} & \text { Thermal conductivity of the tissue, } \mathrm{W} /(\mathrm{m}-\mathrm{K}) \\ k_{s} & \text { Thermal conductivity of the thermistor, } \mathrm{W} /(\mathrm{m}-\mathrm{K}) \\ \text { LDF } & \text { Laser Doppler flowmetry } \\ \text { MEG } & \text { Magnetoencephalography }\end{array}$




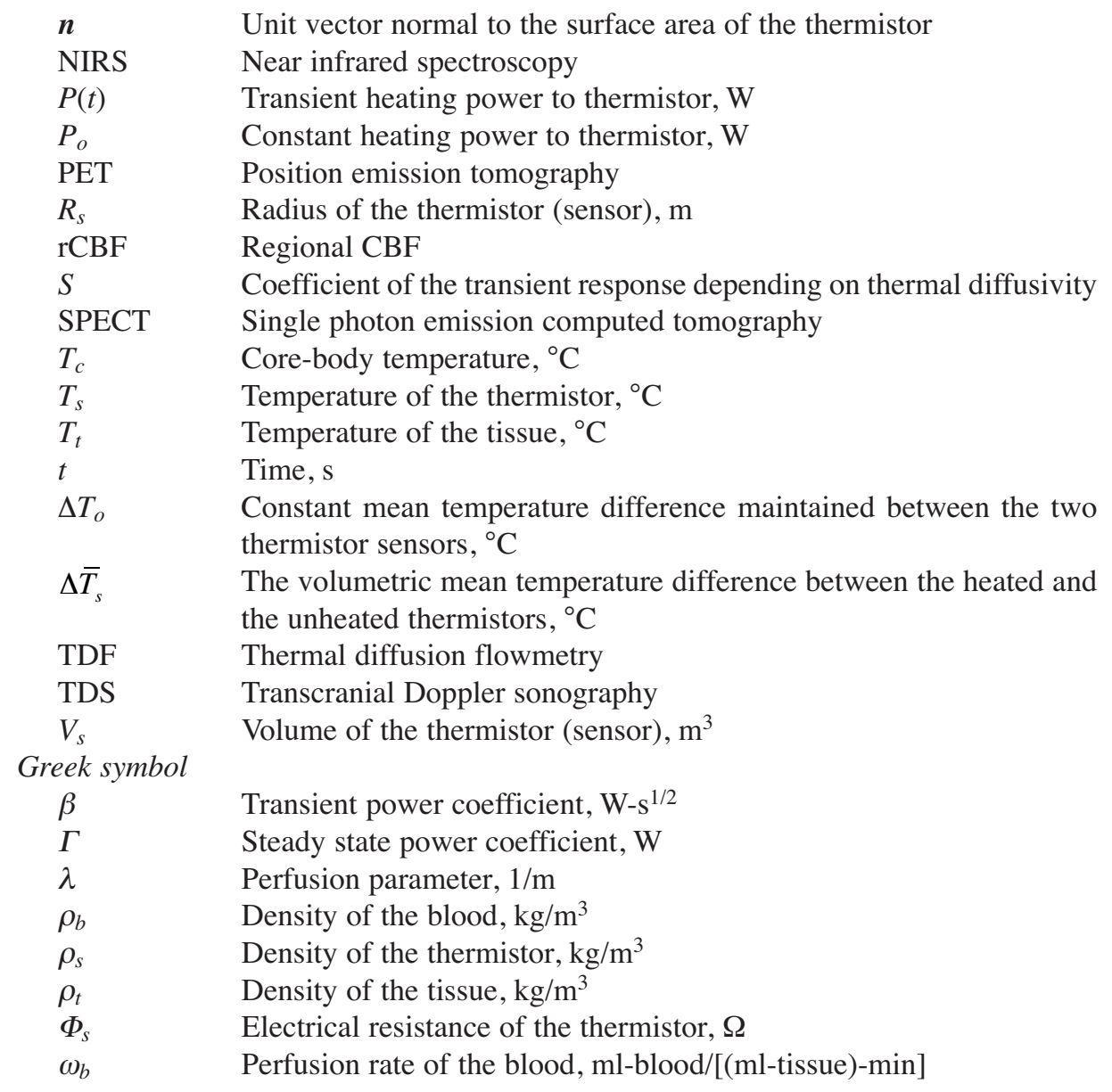

\section{REFERENCES}

[1] Baumgartner C, Serles W, Leutmezer F, Pataraia E, Aull S, Czech T, Pietrzyk U, Relic A, Podreka I. Preictal SPECT in Temporal Lobe Epilepsy: Regional Cerebral Blood Flow is Increased Prior to Electroencephalography-Seizure Onset as process. Medicine, 1998, 39:978-82.

[2] Tyvaert L, Levan P, Dubeau F, Gotman J. Noninvasive Dynamic Imaging of Seizures in Epileptic Patients. Human Brain Mapping, 2009, 4011:3993-4011.

[3] Iasemidis LD. Epileptic seizure prediction and control. IEEE Transactions Biomedical Engineering, 2003, 50:549-558.

[4] Curing the Epilepsies: The Promise of Research, National Institute of Neurological Disorders and Stroke, National Institutes of Health, USA, 2015.

http://www.ninds.nih.gov/disorders/epilepsy/epilepsy_research.htm. Accessed April 2015.

[5] Litt B, Echauz J. Prediction of epileptic seizures. Lancet Neurology, 2002, 1:22-30.

[6] Mormann F, Andrzejak RG, Elger CE, Lehnertz K. Seizure prediction: the long and winding road. Brain, 2007, 130:314-33.

[7] Schelter B, Winterhalder M, Maiwald T, Brandt A, Schad A, Schulze-Bonhage A, Timmer J. Testing statistical significance of multivariate time series analysis techniques for epileptic seizure prediction. Chaos, 2006, 16:013108. 
[8] Cook MJ, O’Brien TJ, Berkovic SF, Murphy M, Morokoff A, Fabinyi G, D’Souza W, Yerra R, Archer J, Litewka L, Hosking S, Lightfoot P, Ruedebusch V, Sheffield WD, Snyder D, Leyde K, Himes D. Prediction of seizure likelihood with a long-term, implanted seizure advisory system in patients with drug-resistant epilepsy: a first-in-man study. The Lancet Neurology, 2013, 12(6), 563-571.

[9] Gadhoumi K, Lina JM, Gotman J. Seizure prediction in patients with mesial temporal lobe epilepsy using EEG measures of state similarity. Clinical Neurophysiology, 2013, 124:1745-1754

[10] Andrzejak RG, Chicharro D, Elger CE, Mormann F. Seizure prediction: any better than chance? Clinical Neurophysiology, 2009, 120:1465-78.

[11] Elger CE, Mormann, F. Seizure prediction and documentation - two important problems, Neurology, 2013, 12:531-532.

[12] Dahl A, Lindegaard K, Russell D, Nyberg-Hansen R, Rootwelt K, Sorteberg W, Nornes H. A comparison of transcranial Doppler and cerebral blood flow studies to assess cerebral vasoreactivity. Stroke, 1992, 23:15-19.

[13] Weinand ME, Carter LP, Patton DD, Oommen KJ, Labiner DM, Talwar D. Long-term surface cortical cerebral blood flow monitoring in temporal lobe epilepsy. Neurosurgery, 1994, 35:657-664.

[14] Weinand ME, Carter LP, El-Saadany WF, Sioutos PJ, Labiner DM, Oommen KJ. Cerebral blood flow and temporal lobe epileptogenicity. Journal of Neurosurgery, 1997, 86:226-32.

[15] Weinand ME, Takacs I, Labiner DM, Ahern GL. Nonepileptic cortical cerebral blood flow and temporal lobe epileptogenicity. Pathophysiology, 1999, 6:135-141.

[16] Weinand ME, Labiner D, Ahern GL. Temporal lobe seizure interhemispheric propagation time depends on nonepileptic cortical cerebral blood flow. Epilepsy research, 2001, 44:33-9.

[17] Gonzalez-Portillo G, Rivero S, Ahern GL, Labiner DM, Weinand ME. Normalization of periictal bihemispheric cerebral perfusion in temporal lobe epilepsy. Pathophysiology, 2004, 11:31-34.

[18] la Fougère C, Rominger A, Förster S, Geisler J, Bartenstein P. PET and SPECT in epilepsy: a critical review. Epilepsy \& Behavior, 2009, 15:50-5.

[19] Carter LP. Thermal diffusion flowmetry. Neurosurgery clinics of North America, 1996, 7:749-54.

[20] Vajkoczy P, Schomacher M, Czabanka M, Horn P. Monitoring Cerebral Blood Flow in Neurosurgical Intensive Care. European Neurological Disease, Issue II, 2007.

[21] Rajan V, Varghese B, van Leeuwen TG, Steenbergen W. Review of methodological developments in laser Doppler flowmetry. Lasers in Medical Science, 2009, 24:269-83.

[22] Krakow K. Imaging epileptic activity using functional MRI. Neurodegenerative Diseases, 2008, 5:286-95.

[23] Laufs H. A personalized history of EEG-fMRI integration. Neurolmage, 2012, 62:1056-1067.

[24] Griffiths PD, Hoggard N, Dannels WR, and Wilkinson D. In vivo measurement of cerebral blood flow: a review of methods and applications. Vascular Medicine, 2001, 6:51-60.

[25] Vongsavan N, Matthews B. Some aspects of the use of laser doppler flow meters for recording tissue blood flow. Experimental Physiology, 1993, 78:1-14.

[26] Kesavadas C, Thomas B. Clinical applications of functional MRI in epilepsy. Indian Journal of Radiology Imaging, 2008, 18:210-217.

[27] Carter LP, White WL, Atkinson JR. Regional cortical blood flow at craniotomy. Neurosurgery, 1978, 2:223-229.

[28] Grayson J. Internal calorimetry in the determination of thermal conductivity and blood flow. Journal of Physiology, 1952, 118:54-72.

[29] Verdú-López F, González-Darder JM, Gonzalez-Lopez P, Macia LB. Using thermal diffusion flowmetry in the assessment of regional cerebral blood flow in cerebral aneurysm microsurgery. Neurosurgery Murcia, 2010, 21:373-80.

[30] Zauner A, Muizelaar JP. Measuring cerebral blood flow and metabolism. Edited by Reilly P, Bullock R. Head Injury. Chapman and Hall Publishing, London, 1997. 
[31] Carter LP, Weinand ME, Oommen KJ. Cerebral blood flow (CBF) monitoring in intensive care by thermal diffusion. Monitoring of Cerebral Blood Flow and Metabolism in Intensive Care. Springer Vienna, 1993, 43-46.

[32] Le Roux PD, Levine J, Kofke WA. Monitoring in Neurocritical Care: Expert Consult: Online. Elsevier Health Sciences, 2013.

[33] Hemedex Inc. BPM Neuromonitoring Guide. 2006-2011.

[34] Bowman HF. U.S. Patent Application 13/999,906, 2014.

[35] Leondes CT. Biomechanical Systems: Techniques and Applications, Volume IV: Biofluid Methods in Vascular and Pulmonary Systems. Vol. 4. CRC Press, 2002.

[36] Idris Z, Mustapha M, Abdullah JM. Neurointensive Care Monitoring for Severe Traumatic Brain Injury, Amit Agrawal (Ed), 2012, 213.

[37] Bowman HF, Newman WH. Method to quantify thermal dissipative mechanisms in biomaterials, U.S. Patent No. 4,852,027. Washington, DC: U.S. Patent and Trademark Office, 1989.

[38] Perl W. Heat and matter distribution in body tissues and the determination of tissue blood flow by local clearance methods. Journal of Theoretical Biology, 1962, 2(3), 201-235.

[39] Wei D, Saidel GM, Jones SC. Optimal Design of a Thermistor Probe for Surface Measurement of Cerebral Blood Flow. IEEE Transactions on Biomedical Eng, 1990, 37:1159-1172.

[40] Chato JC. A Method for the measurement of thermal properties of biologic materials, in Symposium on thermal problems in biotechnology. Chato J.C. (Ed), 1968, 16-25, New York, ASME.

[41] Valvano JW, Allen JT, Bowman HF. The simultaneous measurement of thermal conductivity, thermal diffusivity, and perfusion in small volumes of tissue. Journal of Biomechanical Engineering, 1984, 106(3), 192-197.

[42] Martin GT, Bowman HF. Validation of real-time continuous perfusion measurement. Medical and Biological Engineering and Computing, 2000, 38(3), 319-325.

[43] Bowman HF, Balasubramaniam TA. A new technique utilizing thermistor probes for the measurement of thermal properties of biomaterials. Cryobiology, 1976, 13(5), 572-580.

[44] Balasubramaniam TA, Bowman HF. Thermal conductivity and thermal diffusivity of biomaterials: A simultaneous measurement technique. Journal of Biomechanical Engineering, 1977, 99(3), 148-154.

[45] Valvano JW. Bioheat transfer. Encyclopedia of Medical Devices and Instrumentation. Webster, JG (Ed), John Wiley \& Sons, Inc., 2006.

[46] Patel PA, Valvano JW, Pearce JA, Prahl SA. A self-heated thermistor technique to measure effective thermal properties from the tissue surface. Journal of Biomechanical Engineering, 1987, 109, 330-335.

[47] Chen MM, Holmes KR. The thermal pulse-decay method for simultaneous measurement the thermal conductivity and local blood perfusion rate of living tissues. Advances in Bioengineering, 1980, 113-115.

[48] PENNES H. Analysis of tissue and arterial blood temperatures in the resting human forearm. Journal of Applied Physiology, 1948, 1(2), 93-122.

[49] Wei D, Saidel GM, Jones SC. Thermal method for continuous measurement of cerebral perfusion. Med. and Biol. Eng. and Computer, 1994, 32:481-488.

[50] Foerster O. Operative Behandlung des Torticollis spastica, Zentralbl. Chir. 1926, 44:2804-2805.

[51] Penfield W. The Evidence for a Cerebral Vascular Mechanism in Epilepsy. Annals of Internal Medicine, 1933, 7:303.

[52] Gibbs FA. A Thermoelectric Blood Flow Recorder in the Form of a Needle. Experimental Biology and Medicine, 1933, 31:141-146.

[53] Gibbs FA, Lennox WG, Gibbs EL. Cerebral blood flow preceding and accompanying epileptic seizures in man. Arch Neurology Psychiatry, 1934, 32:257-272.

[54] Penfield W. Cerebral Blood Flow during Induced Epileptiform Seizures in Animals and Man. Journal of Neurophysiology, 1939, 2:257. 
[55] Penfield W. Remarks on incomplete hypotheses for the control of cerebral circulation. Journal of Neurosurgery, 1971, 35:124-127.

[56] Brawley BW. The pathophysiology of intracerebral steal following carbon dioxide inhalation, an experimental study. Scand J Clin Lab Invest. 1968, 22:102.

[57] Carter LP, Atkinson JR. Cortical blood flow in controlled hypotension as measured by thermal diffusion. Journal of Neurology, Neurosurgery, and Psychiatry, 1973a, 36:906-991.

[58] Carter LP, Atkinson JR. Auto-regulation and Hyperemia of Cerebral Blood Flow as Evaluated by Thermal Diffusion. Stroke, 1973b, 4:917-922.

[59] Carter LP, Erspamer R, Bro W. Cortical blood flow: thermal diffusion vs isotope clearance. Stroke, 1981, 12:513-518.

[60] Oommen KJ, Weinand M, Carter L. Cerebral Blood Flow over the Epileptogenic Cortex, Before, During and After Seizure. Epilepsia, 1993, 34:127-128 (abstract).

[61] Federico P, Abbott DF, Briellmann RS, Harvey S, Jackson GD. Functional MRI of the pre-ictal state. Brain: a Journal of Neurology, 2005, 128:1811-7.

[62] Oommen KJ, Saba S, Oommen JA, Francel PC, Arnold CD, Wilson DA. The Relative Localizing Value of Interictal and Immediate Postictal SPECT In Seizures of Temporal Lobe Origin, J Nucl Med., 2004, 45:2021-2025.

[63] Oommen KJ, Oommen JA, Lie DYC, Imam S, Chyu M, Zhang Y. Relationship between Cerebral Perfusion and EEG in the Rat Brain. Int IEEE EMBS Conf Neural Eng, 2011, 174-177.

[64] Aarabi A, Fazel-Rezai R, Aghakhani Y. EEG seizure prediction: measures and challenges. Conf Proc IEEE Eng Med Biol Soc, 2009, 1864-7.

[65] Arthurs S, Zaveri HP, Frei MG, Osorio I. Patient and caregiver perspectives on seizure prediction. Epilepsy \& Behavior, 2010, 19(3), 474-477.

[66] Christopher CJ, Gregory KB. Characterization of early partial seizure onset: Frequency, complexity and entropy. Clinical Neurophysiology, 2012, 123:658-669.

[67] Gotman J, Kobayashi E, Bagshaw AP, Bénar CG, Dubeau F. Combining EEG and fMRI: a multimodal tool for epilepsy research. Journal of Magnetic Resonance Imaging, 2006, 23:906-20.

[68] Moghim N, David WC. Predicting Epileptic Seizures in Advance. PloS one 9.6, 2014, e99334.

[69] Bandarabadi M, Teixeira CA, Rasekhi J, Dourado A. Epileptic seizure prediction using relative spectral power features. Clinical Neurophysiology, 2015, 126(2), 237-248.

[70] Snyder DE, Echauz J, Grimes DB, Litt B. The statistics of a practical seizure warning system. Journal of Neural Engineering, 2008, 5(4), 392.

[71] Ramgopal S, Thome-Souza S, Jackson M, Kadish NE, Fernández IS, Klehm J, Bosl W, Reinsberger C, Schachter S, Loddenkemper T. Seizure detection, seizure prediction, and closed-loop warning systems in epilepsy. Epilepsy \& Behavior, 2014, 37, 291-307.

[72] Winterhalder M, Maiwald T, Voss HU, Aschenbrenner-Scheibe R, Timmer J, Schulze-Bonhage A. The seizure prediction characteristic: a general framework to assess and compare seizure prediction methods. Epilepsy \& Behavior, 2003, 4(3), 318-325.

[73] Schulze-Bonhage A, Sales F, Wagner K, Teotonio R, Carius A, Schelle A, Ihle M. Views of patients with epilepsy on seizure prediction devices. Epilepsy \& Behavior, 2010, 18(4), 388-396.

[74] Sackellares JC. Seizure prediction and monitoring. Epilepsy Behavior, 2010, 18:106-109. 


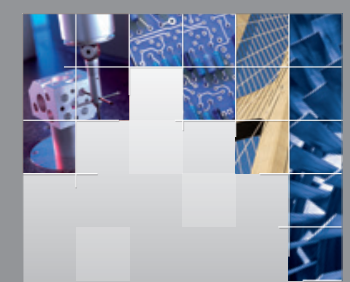

\section{Enfincering}
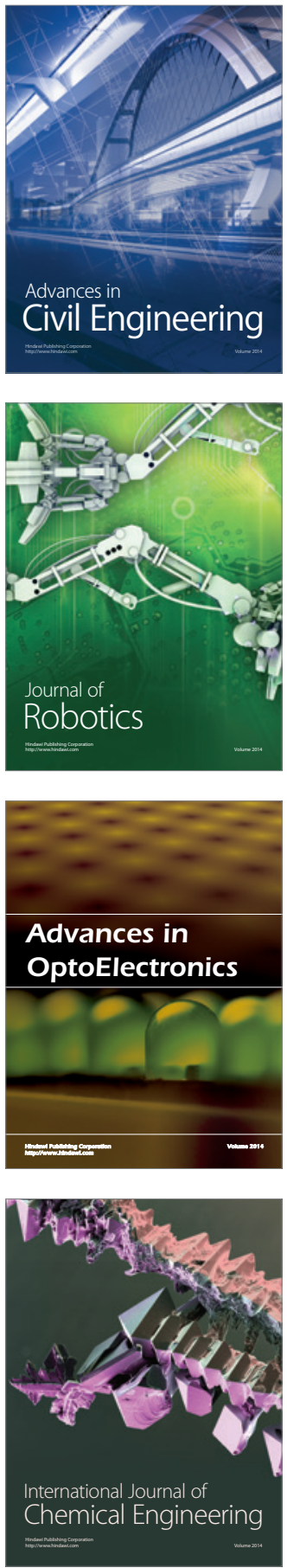

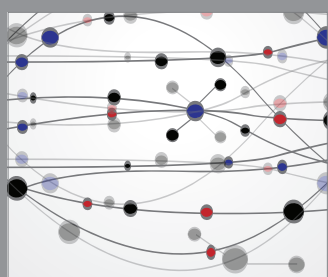

The Scientific World Journal

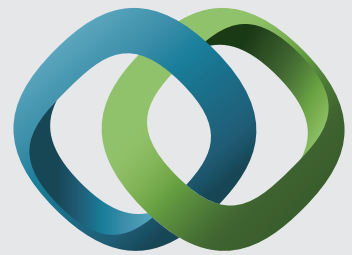

\section{Hindawi}

Submit your manuscripts at

http://www.hindawi.com
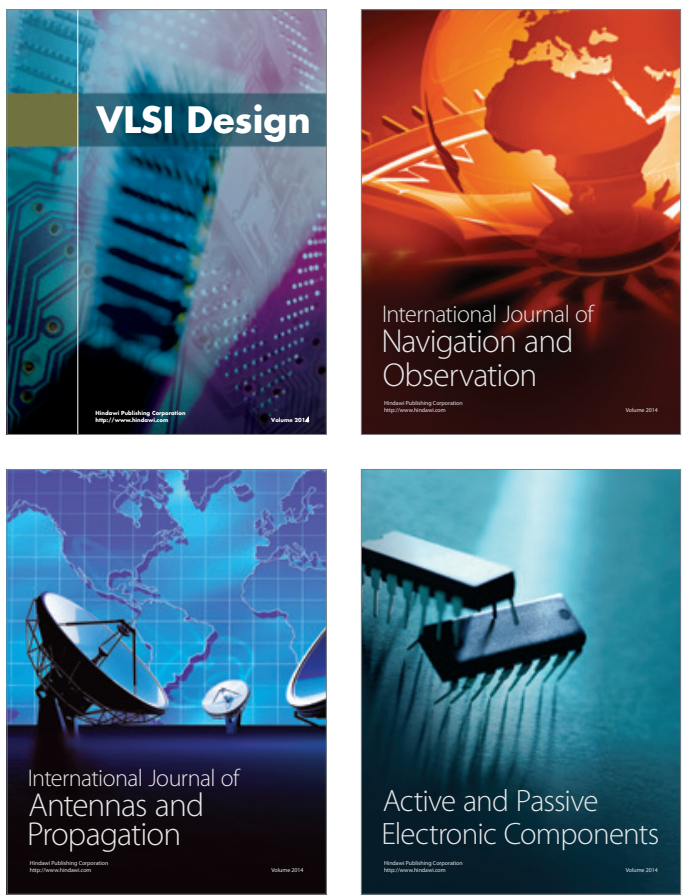
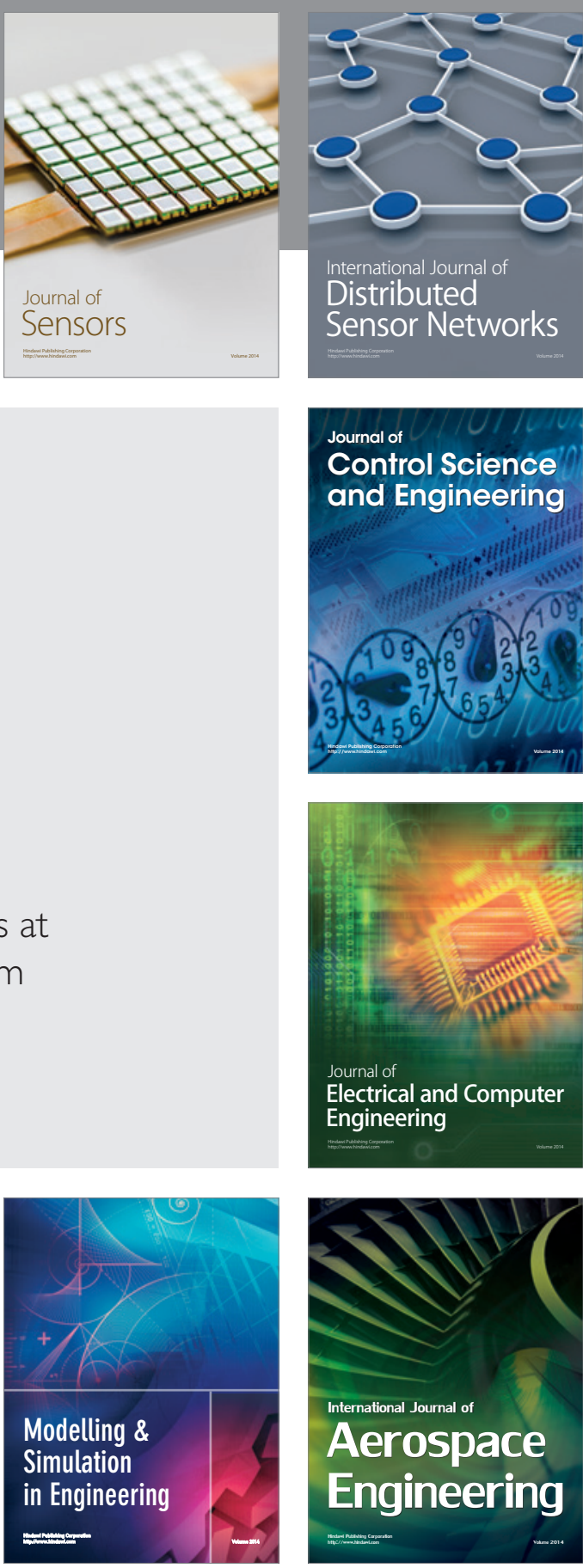

International Journal of

Distributed

Sensor Networks

Journal of

Control Science

and Engineering
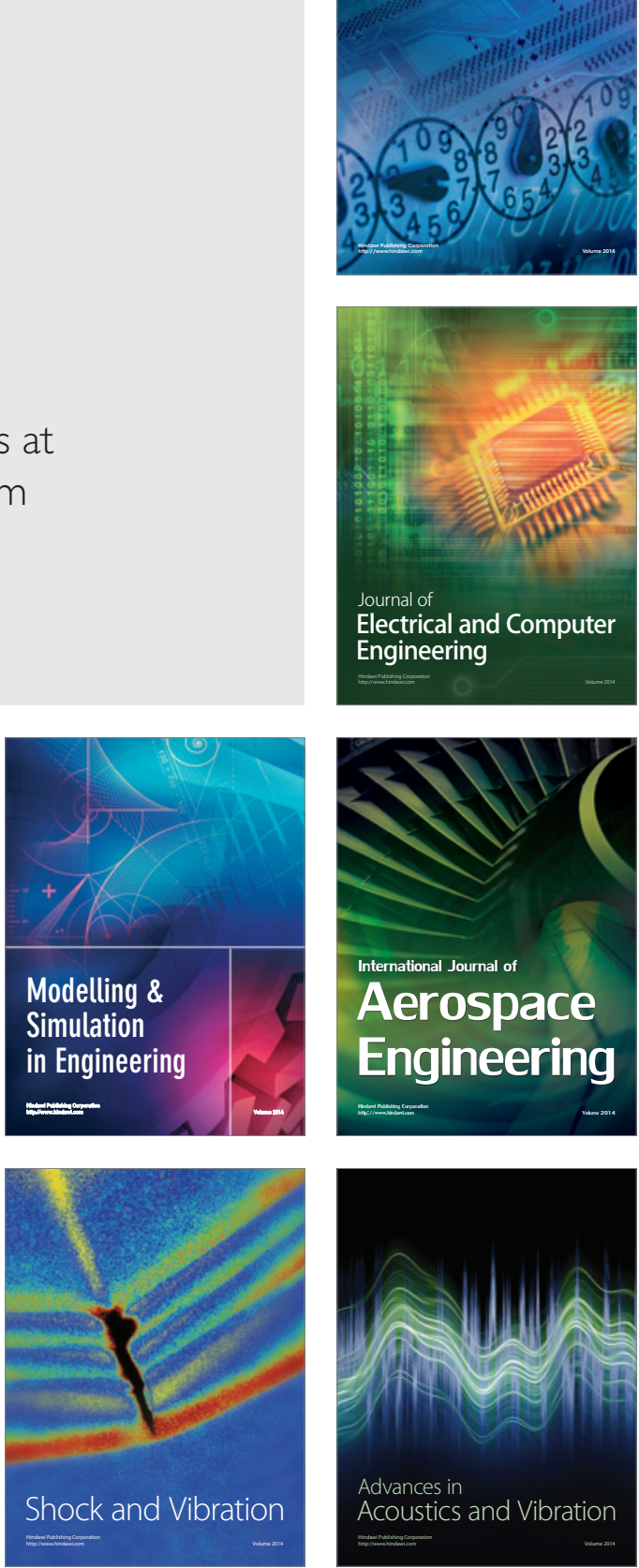